\title{
Impact of occultations of stellar active regions on transmission spectra
}

\section{Can occultation of a plage mimic the signature of a blue sky?}

\author{
M. Oshagh ${ }^{1,2,3}$, N. C. Santos ${ }^{1,2,3}$, D. Ehrenreich ${ }^{4}$, N. Haghighipour ${ }^{5}$, P. Figueira ${ }^{1,3}$, A. Santerne ${ }^{1,3}$, and M. Montalto ${ }^{1,3}$ \\ ${ }^{1}$ Centro de Astrofísica, Universidade do Porto, Rua das Estrelas, 4150-762 Porto, Portugal \\ e-mail: moshagh@astro.up.pt \\ 2 Instituto de Astrofísica e Ciências do Espaço, Universidade do Porto, CAUP, Rua das Estrelas, 4150-762 Porto, Portugal \\ 3 Departamento de Física e Astronomia, Faculdade de Ciências, Universidade do Porto, Rua do Campo Alegre, $4169-007$ Porto, \\ Portugal \\ ${ }^{4}$ Observatoire de Genève, Université de Genève, 51 chemin des Maillettes, 1290 Sauverny, Switzerland \\ 5 Institute for Astronomy and NASA Astrobiology Institute, University of Hawaii-Manoa, 2680 Woodlawn Drive, \\ Honolulu HI 96822, USA
}

Received 24 April 2014 / Accepted 8 July 2014

\begin{abstract}
Transmission spectroscopy during planetary transits, which is based on the measurements of the variations of the planet-to-star radius ratio as a function of wavelength, is a powerful technique to study the atmospheric properties of transiting planets. One of the main limitations of this technique is the effects of stellar activity, which up until now, have been taken into account only by assessing the effect of non-occulted stellar spots on the estimates of the planet-to-star radius ratio. In this paper, we study the impact of the occultation of a stellar spot and plage on the transmission spectra of transiting exoplanets for the first time. We simulated this effect by generating a large number of transit light curves for different transiting planets, stellar spectral types, and different wavelengths. Results of our simulations indicate that the anomalies inside the transit light curve can lead to a significant underestimation or overestimation of the planet-to-star radius ratio as a function of wavelength. At short wavelengths, the effect can reach to a difference of up to $10 \%$ in the planet-to-star radius ratio, mimicking the signature of light scattering in the planetary atmosphere. Atmospheric scattering has been proposed to interpret the increasing slopes of transmission spectra toward blue for exoplanets HD 189733b and GJ 3470b. Here, we show that these signatures can be alternatively interpreted by the occultation of stellar plages. Results also suggest that the best strategy to identify and quantify the effects of stellar activities on the transmission spectrum of a planet is to perform several observations during the transit epoch at the same wavelength. This will allow for identifying the possible variations in transit depth as a function of time due to stellar activity variability.
\end{abstract}

Key words. planets and satellites: atmospheres - techniques: photometric - methods: data analysis - methods: numerical stars: activity

\section{Introduction}

Most attempts in characterizing exoplanetary atmospheres have been made by using the transmission spectroscopy (multiband photometry) of transiting exoplanets. In this approach, observations of planetary transits in different wavelengths are used to determine planet-to-star radius ratio, $R_{\mathrm{p}} / R_{*}=k(\lambda)$, as a function of color. The inferred wavelength dependence of this quantity is the result of differential absorption in the planetary atmosphere (Seager \& Sasselov 2000; Brown et al. 2001; Charbonneau et al. 2002) which has been used to constrain different atmospheric composition models: for example GJ 3470b (Demory et al. 2013; Fukui et al. 2013; Nascimbeni et al. 2013; Crossfield et al. 2013), GJ 1214b (Kreidberg et al. 2014), HD 209458b (Désert et al. 2008), and HD 189733b (Pont et al. 2007, 2008, 2013; Ehrenreich et al. 2007; Sing et al. 2009, 2011).

Although the magnitude of the planet-to-star radius ratio is strongly affected by the activity of the central star and its corresponding surface features (Czesla et al. 2009; Oshagh et al. 2013a), except for the systems of GJ436, GJ3470, and HD 189733 (Pont et al. 2007, 2013; Knutson et al. 2011; Sing et al. 2011; Nascimbeni et al. 2013), in most studies using transmission spectroscopy, the impact of stellar features has not been taken into account. Even in the cases of GJ436 and GJ3470, only the impact of non-occulted stellar spots has been discussed. The system of HD 189733 is the only case in which the effect of the occulted stellar spot inside the transit has been taken into account.

The main objective of this paper is to examine the possible impact of the occultation of stellar activity features (such as spots and plages) by transiting planets, on transmission spectra. In particular and for the first time, we explore the impact of the occultation of plages (bright regions in the stellar chromosphere). We perform a large number of simulations to quantify the impact of this effect on the transit depth measurements in different wavelengths and for various physical configurations. To demonstrate the application of our results, we apply our methodology to the planetary systems of two active stars HD 189733 and GJ 3470. Due to their low density and large planet-to-star radius ratio, these systems are among the most favorable targets for atmospheric characterization purposes. The measurements of planet radii in these systems have shown an excess in the short wavelength regime of 300-800 $\mathrm{nm}$. Several studies of the entire transmission spectra of these two planets have contributed those 
observed excesses to Rayleigh scattering processes in the planets' atmospheres. In this paper, we investigate the possibility of explaining these excesses features by taking the effects of stellar spots and plages occultation into account.

In Sect. 2, we present the details of our models that are used to produce light curves of a system which has a planet transiting plages and spots on the surface of its host star. In Sect. 3, we apply our models to different configurations of stars with different spectral types, stellar activity features, and for planet radii corresponding to wavelengths ranging from $400 \mathrm{~nm}$ to $4500 \mathrm{~nm}$. In Sect. 4 we reanalyze the transmission spectra of HD 189733b as reported by Pont et al. (2013) and GJ 3470b as reported by Nascimbeni et al. (2013), and explore possible scenarios which could reproduce the same transmission spectra by invoking only occultation of stellar plages. In Sect. 5, we conclude our study by summarizing the results and discussing their implications.

\section{Description of the model}

We considered a transiting system with a planet in a 3 day orbit. We chose the central star to be of spectral types $M$ and $G$ and took the planet to be of Jupiter $(\mathrm{J})$ and Neptune $(\mathrm{N})$ sizes. The $\mathrm{G}$ star was chosen to be Sun-like and the radius of the M star was taken to be 0.7 solar-radii. Both stars have a rotational period of 9 days. The planet-to-star radius ratios in these systems are $0.035,0.1,0.05$, and 0.15 for the NG, JG, NM, and JM systems, respectively. We chose these systems because the large values of their planet-to-star radius ratio make them favorable for planet atmosphere studies.

To study the effects of spots and plages, we assigned them a filling factor defined as

$$
f=\frac{A_{\mathrm{s}}}{A_{*}}=\left(\frac{R_{\mathrm{S}}}{R_{*}}\right)^{2} .
$$

In this equation, $A_{\mathrm{S}}$ is the area of the stellar activity feature, $R_{\mathrm{S}}$ is its radius, and $A_{*}$ is is the area of the stellar disk. In applying Eq. (1) to spots, we considered $f$ to be $0.25 \%$ and $1 \%$ where $1 \%$ is the largest filling factor of a Sun spot (Solanki 2003; Meunier et al. 2010). For plages, we considered $f=0.25 \%, 1 \%$, and $6.25 \%$, in which the maximum value coincides with the maximum filling factor of a Sun's plage (Meunier et al. 2010). At the time of the overlap between the transiting planet and the stellar activity feature, we assumed that the feature is on the star's equator and at a longitude equal to 0.5 stellar radii. As shown by Oshagh et al. (2013a), the maximum underestimation of the planet radius in the transit light curve with a spot anomaly inside the transit occurs when the spot anomaly appears in the center of the transit light curve.

\subsection{Generating light curves}

To generate the light curves of our system, we used the publicly available software SOAP-T (Oshagh et al. 2013b). This software has the capability of producing light curves of systems where a transiting planet orbits a rotating star with activity features in the form of stellar spots or plages. The three wavelength-dependent stellar parameters in this software, namely the coefficients of quadratic stellar limb darkening $\left(u_{1}\right.$ and $\left.u_{2}\right)$ and the relative brightness of stellar active regions $(b)$ enable one to produce light curves for different values of wavelength. Table 1 shows the values of $u_{1}$ and $u_{2}$ adopted from the catalog by Claret \& Bloemen (2011) for the values of the wavelength used in our
Table 1. Quadratic limb darkening coefficients for $M$ and $G$ stars in different wavelength (Claret \& Bloemen 2011).

\begin{tabular}{lccccc}
\hline \hline$\lambda(\mathrm{nm})$ & 400 & 800 & 1500 & 3000 & 4500 \\
\hline M-dwarf & & & & & \\
$u_{1}$ & 0.45 & 0.43 & 0.40 & 0.05 & 0.05 \\
$u_{2}$ & 0.36 & 0.34 & 0.36 & 0.20 & 0.18 \\
\hline G-dwarf & & & & & \\
$u_{1}$ & 0.70 & 0.30 & 0.10 & 0.07 & 0.05 \\
$u_{2}$ & 0.18 & 0.30 & 0.32 & 0.14 & 0.12 \\
\hline
\end{tabular}

simulations. These wavelengths have been chosen to cover the entire visible spectrum and the near- and mid-infrared ranges.

To determine the value of $b$, we note that this quantity can be written as

$$
b(\lambda)=\frac{\exp \left[h c / \lambda K_{\mathrm{B}} T_{*}\right]-1}{\exp \left[h c / \lambda K_{\mathrm{B}} T_{\mathrm{s}}\right]-1}
$$

to the first-order of approximation. In this equation, $h$ is the Planck constant, $c$ is the speed of light, $K_{\mathrm{B}}$ is the Boltzmann constant, $T_{*}$ is the stellar temperature, and $T_{\mathrm{S}}$ denotes the temperature of the activity feature. We considered $T_{*}=5780 \mathrm{~K}$ and $3000 \mathrm{~K}$ for our $\mathrm{G}$ and $\mathrm{M}$ stars, respectively. For any star with a temperature within this range, the amplitude of the effect of the occultation of its active region is between that of these two stars (Fig. 3).

To obtain the value of $T_{\mathrm{s}}$ for a stellar spot, we used Fig. 7 of Berdyugina (2005), where the observed temperature of spots for stars with different spectral types is presented. For plages, this temperature has only been measured for the Sun (Worden et al. 1998; Unruh et al. 1999; Meunier et al. 2010), which showed the maximum temperature contrast of $300 \mathrm{~K}$ for a plage at the Sun's limb and the minimum of $100 \mathrm{~K}$ at the center of the Sun. We note that the spots on the surface of the Sun show a limbdarkening effect. That is, a Sun spot at the center of the Sun's disk shows a higher contrast compared to the one on the Sun's limb (Unruh et al. 1999; Meunier et al. 2010). On the contrary, a Sun plage shows a limb brightening behavior, which means that a Sun plage on the Sun's limb displays higher contrast compared to that on the center of the Sun's disk (Meunier et al. 2010). The values for the temperature of spots and plages in this study have been chosen by considering this opposite behavior of the spot's limb darkening and the plage's limb brightening. This enabled us to consider the impact of the position of a stellar spot/plage on its maximum temperature-contrast when simulating our systems. For instance, a stellar spot in the center of the stellar disk could reach the maximum possible temperature-contrast with the stellar photosphere, whereas a plage in that location would reach only a third of the maximum possible plage temperaturecontrast. By taking into account that in our simulations, we consider that the plages are located at the center of the stellar disk, therefore, we use a $100 \mathrm{~K}$ temperature contrast for a plage on the surface of our G star, and by assuming that on an M star, a plage at its maximum temperature contrast will have a similar temperature-difference. Table 2 shows the values of $T_{\mathrm{s}}$ for all our models. The variations of $b(\lambda)$ as a function of the wavelength is shown in Fig. 1.

\section{Results of simulations}

We generated a large number of mock transit light curves for our $\mathrm{G}$ and $\mathrm{M}$ stars by considering transiting planets with radii equal to those of Neptune and Jupiter. As we are interested in 
Table 2. Detailed parameters of our models.

\begin{tabular}{lcccc}
\hline \hline Model number & $T_{*}$ & $T_{\mathrm{s}}$ & $f$ & $R_{\mathrm{p}} / R_{*}$ \\
\hline G dwarf+ spot & & & & \\
1 & 5780 & 4000 & $0.25 \%$ & 0.035 \\
2 & 5780 & 4000 & $0.25 \%$ & 0.1 \\
3 & 5780 & 4000 & $1 \%$ & 0.035 \\
4 & 5780 & 4000 & $1 \%$ & 0.1 \\
\hline G dwarf+ plage & & & & \\
5 & 5780 & 5880 & $0.25 \%$ & 0.035 \\
6 & 5780 & 5880 & $0.25 \%$ & 0.1 \\
7 & 5780 & 5880 & $1 \%$ & 0.035 \\
8 & 5780 & 5880 & $1 \%$ & 0.1 \\
9 & 5780 & 5880 & $6.25 \%$ & 0.035 \\
10 & 5780 & 5880 & $6.25 \%$ & 0.1 \\
\hline M dwarf + spot & & & & \\
11 & 3000 & 2500 & $0.25 \%$ & 0.05 \\
12 & 3000 & 2500 & $0.25 \%$ & 0.15 \\
13 & 3000 & 2500 & $1 \%$ & 0.05 \\
14 & 3000 & 2500 & $1 \%$ & 0.15 \\
\hline M dwarf + plage & & & & \\
15 & 3000 & 3100 & $0.25 \%$ & 0.05 \\
16 & 3000 & 3100 & $0.25 \%$ & 0.15 \\
17 & 3000 & 3100 & $1 \%$ & 0.05 \\
18 & 3000 & 3100 & $1 \%$ & 0.15 \\
19 & 3000 & 3100 & $6.25 \%$ & 0.05 \\
20 & 3000 & 3100 & $6.25 \%$ & 0.15 \\
\hline
\end{tabular}

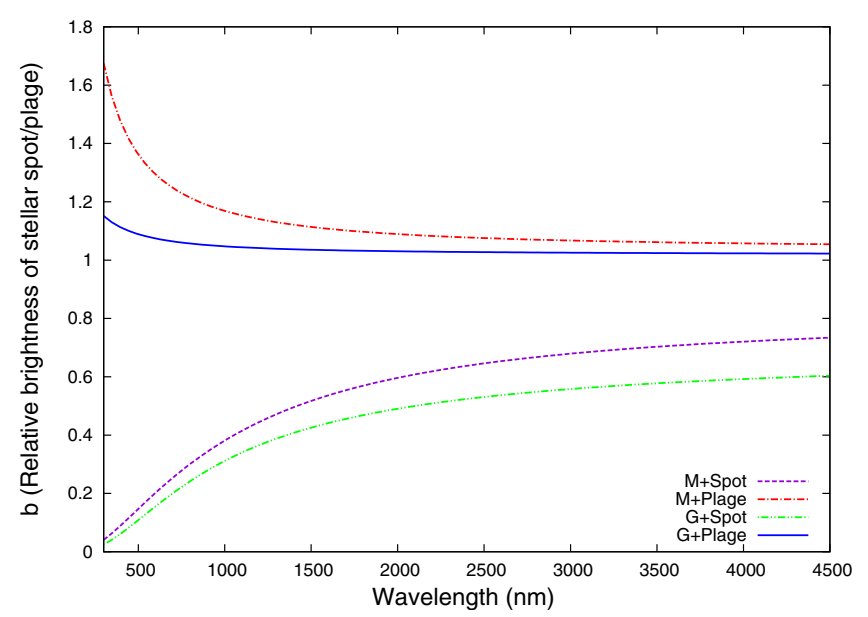

Fig. 1. Relative brightness of stellar spots and plages on $M$ and $G$ stars as a function of wavelength. The details of the parameters of spots, plage, and stars are listed in Table 2.

determining the impact of the occultation of stellar active regions on the estimates of the radius of the transiting planet, we considered our stars to contain plages or spots and generated light curves for different values of wavelength (see Table 1). As a result, all the generated mock light curves exhibited anomalies in their transit parts. To examine the effects of these anomalies on the estimates of the planet radius, we fit all these mock light curves with transit light curve models corresponding to systems in which the planet does not occult the stellar active region. In each fitted model, we set all the parameters equal to those used to generate the system's initial mock light curve, except for the planet's radius, which we allowed to vary freely. In general, the latter curves show the exact same behavior as those with anomalies, except for inside the transit where the spot/plage occulted anomalies exist. Figure 2 shows a sample of our fitting results (model 16 in Table 2). The light curve in red corresponds to the
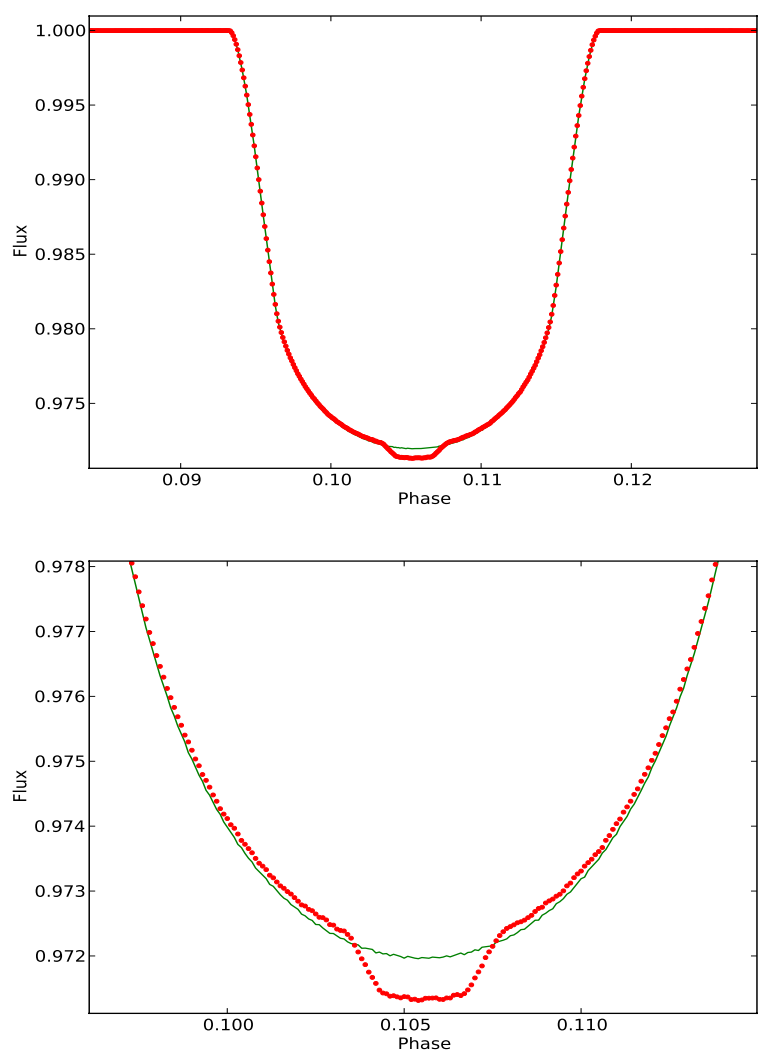

Fig. 2. Top: a sample light curve of our simulations. The system consists of an $\mathrm{M}$ star with a 9 day rotational period with a Jupiter-sized planet $\left(R_{\mathrm{p}} / R_{*}=0.15\right)$ in a 3 day orbit. We considered a plage with a filling factor of $0.25 \%$ on the surface of the star. The red curve corresponds to the case when the transiting planet occults the plage. The green curve shows the best fit transit light curve without considering the occultation. Bottom: zoomed-in at the bottom of light curve.

system in which the planet occulted a stellar plage. The green curve represents the best-fitted transit without the occultation of that plage.

It is important to mention that during the fitting procedure, we allow the depth of the transit to vary as a free parameter, while holding other parameters of the system constant. In that case the best fit anomaly-free light curve is used to determine the best planet-to-star radius ratio for each light curve with inside transit anomalies. It should be noted that only part of the spot/plage would be covered by the planet in systems where the planet radius is smaller than the radius of the stellar active region (for instance, model 3 in Table 2) and as a result, the remaining fraction of the spot/plage will affect the out-of-transit light curve similar to an un-occulted spot/plage. However, as explained above, in our simulations, the only difference between the light curve containing an anomaly and the anomaly-free light curve is only inside their transits, this out-of-transit light will not affect the results of our fitting.

Figure 3 shows the relative error in the estimate of the planetary radius obtained from the anomaly-free fitting procedure compared to the radius of the planet that is used in generating the mock light curves (i.e., Neptune or Jupiter radii). As shown here, the underestimation or overestimation of the planet-to-star radius ratio can be quite high $(\sim 10 \%)$, particularly on the blue side of the spectrum where the stellar active regions show higher contrast. These results also suggest that the stellar spot/plage filling factor seems to play an important role in the estimate of the planet's radius. For instance, the maximum effect appears for 

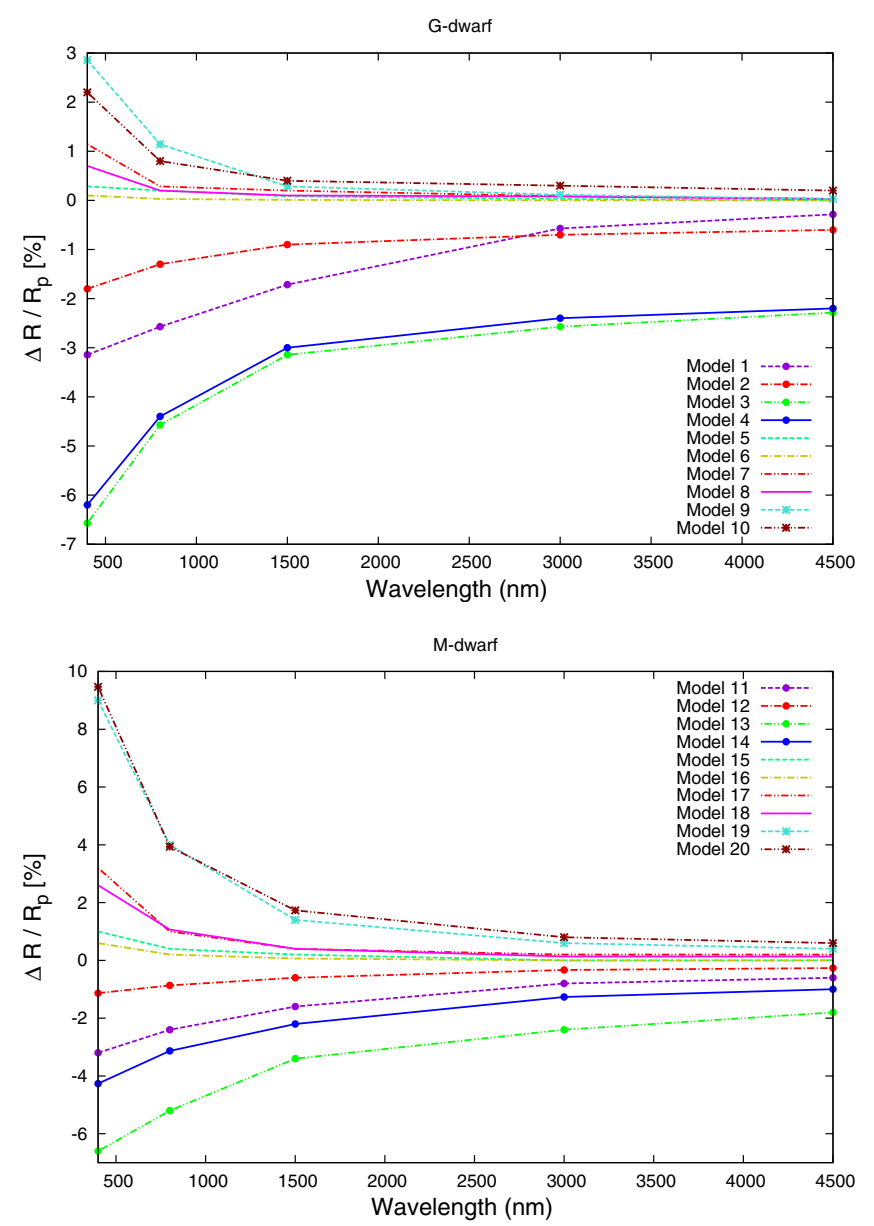

Fig. 3. Relative error in the estimate of the radius of a transiting planet as a function of wavelength calculated by comparing the value of the planet radius obtained from the best-fitted anomaly-free light curve with that obtained from the light curve by taking the effects of stellar spots and plages occultation into account. Different colors correspond to different models, as described in Table 2.

the largest filling factors corresponding to models 3, 4, 9 and 10 in the models where a Jupiter- or Neptune-sized planet orbit a G-dwarf (see Table 2). It should be noted that the temperature contrast between a stellar active region and its surrounding area and the value of the filling factor impose a strong degeneracy to the estimate of the planet's radius. That is, a variation in the temperature contrast of a spot or plage can be compensated by a properly chosen value of the filling factor, such that different combinations of temperature contras/filling factor produce the same value for the planet's radius.

As we noted earlier in all our simulations, we assumed that the stellar rotation axis is parallel to the plane of sky, the orbit of the transiting planet is edge-on, and the occultation of a spot/plage occurs when this feature is on the center of the star's disk. As a result of these assumptions, the values presented here are upper limits.

\section{Reanalyzing HD 189733b and GJ 3470b: Do they have blue skies?}

\section{1. $H D 189733 b$}

HD 189733 is a K-dwarf with an effective temperature of $5050 \mathrm{~K}$, surface gravity of $\log g=4.53$, and brightness of $V \simeq 7.7$ (Sing et al. 2011). The short-period planet of this star,

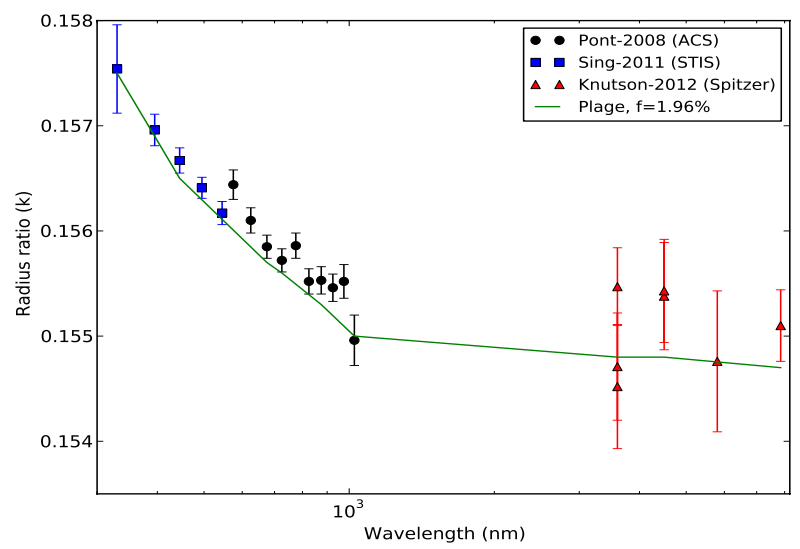

Fig. 4. Observed transmission spectrum of HD 189733b and reconstructed transmission spectrum by assuming the planet overlapping a stellar plage. The excess in the estimate of the planet radius-ratio in the blue side of spectrum can be reproduced by presuming a plage on the surface of HD 189733. See text for more details.

HD 189733b, is a Jupiter-like body in a 2.2 day orbit with a scaled semimajor axis of $a / R_{*}=8.92$ (Pont et al. 2007). The ratio of the radius of HD $189733 \mathrm{~b}$ to that of its host star is 0.155 . The large atmospheric scale height of HD $189733 \mathrm{~b}$ has made this planet one of the best studied systems in the studies of exoplanetary atmospheres (e.g., Lecavelier Des Etangs et al. 2008; Pont et al. 2008, 2013; Sing et al. 2011). As reported by Sing et al. (2011) and Pont et al. (2013), HD 189733b shows an excess in its radius in the entire visual band (300-800 nm), which can be attributed to Rayleigh scattering in the planet's atmosphere.

The star HD 189733 is a highly active star. It has a strong $\mathrm{X}$-ray emission and intense chromospheric Ca II H and $\mathrm{K}$ lines, which suggest a high level of activity on the surface of this star (Knutson et al. 2010; Poppenhaeger et al. 2013). During its 12 day stellar rotation, HD 189733 also shows photometric modulation $\left(\right.$ Photo $_{\text {var }}$ ) of up to $\simeq 2 \%$ in the mean $b$ and $y$ Strömgren bands (510 nm) (Boisse et al. 2009; Sing et al. 2011). Sing et al. (2011) have shown that the light curve of HD 189733 carries clear signatures of stellar spot occultation inside its transit. These authors and, more recently, Pont et al. (2013) studied the effects of occulted and non-occulted stellar spots on the estimates of the radius of HD 189733b and found that these anomalies have significant effects on the accurate measurements of the planet's radius.

We examined the possible influences of stellar plages on the spectra of HD 189733b and the measurement of its radius. Following the procedure described in Sect. 3, we generated mock light curves of HD 189733 for different values of wavelength by considering an overlap between a plage and the transiting planet. We then fitted these mock light curves with models in which the planet/plage occultation was not taken into account. Results of our analysis indicated that the observed transmission spectrum of HD $189733 \mathrm{~b}$ can be reproduced by considering the planet that occults a stellar plage with a filling factor of $1.96 \%$ and a temperature-contrast of $100 \mathrm{~K}$. As shown in Fig. 4, the observed excess in the planet radius in the bluer part of spectrum is also well matched by the predictions of the plage-occultation scenario.

By considering the above filling factor and the relative brightness of a plage as obtained from Eq. (2), we found that the amplitude of the photometric modulations of HD 189733 is approximately $0.4 \%$ at $510 \mathrm{~nm}$ wavelength, which is noticeably smaller than the $\sim 2 \%$ value obtained from observation. 
This suggests that our proposed scenario (i.e., the occultation of a plage on the surface of the star) is physically viable. We note here that as explained before, determining the plage's temperature and filling factor introduce a strong degeneracy in the modeling process. For instance, if we considered a temperature ratio of $\sim 0.87$ for HD 189733 compared to the Sun and used this ratio to estimate the temperature contrast of a plage on the surface of HD 189733 ( $\sim 65 \mathrm{~K})$, the required plage's filling factor for reproducing the transmission spectra of HD 189733b would be around $2.89 \%$.

\subsection{GJ $3470 b$}

The star GJ 3470 is an M-star with a temperature of $3600 \mathrm{~K}$ and surface gravity of $\log g=4.658$ (Demory et al. 2013). This star is host to a Uranus-mass planet (GJ 3470b) in a 3.34 day orbit with a scaled semimajor axis of $a / R_{*}=14.02$ (Fukui et al. 2013) and a planet-to-star radius ratio of 0.078 . The planet GJ $3470 \mathrm{~b}$ was first discovered with the radial velocity technique using the HARPS spectrograph. The transits of this planet were later detected through ground-based follow-up observation by Bonfils et al. (2012). Recently, several attempts were made to characterize the atmosphere of GJ 3470b using ground- and space-based facilities (Fukui et al. 2013; Demory et al. 2013; Crossfield et al. 2013; Nascimbeni et al. 2013; Ehrenreich et al. 2014). These observations indicated that the planet's atmosphere has a flat infrared spectrum between 1 and $5 \mu \mathrm{m}$, suggesting an increase in slope toward the blue side of spectrum $(\lambda \sim 360 \mathrm{~nm})$, which was interpreted by Nascimbeni et al. (2013) to be the result of Rayleigh scattering in the atmosphere.

As suggested by Bonfils et al. (2012) GJ 3470 may not be a very active star because of its slow rotation. Fukui et al. (2013) confirmed this finding by using photometric observations and stated that thus star may not be very active, although GJ 3470 showed photometric variability of approximately Phot $_{\mathrm{var}} \simeq 1 \%$ at Ic-band $(786.5 \mathrm{~nm})$, during the course of their 60-day observations. Those variabilities can be explained by assuming that GJ 3470 harbors a spot with a filling factor of $1 \%$. The latter motivated Nascimbeni et al. (2013) to study the possible contribution of a non-occulted stellar spot on the spectrum of GJ 3470b, and they concluded that their results are not affected by that effect. In this section, we examine whether an occulted stellar plage can affect the results.

We generated synthetic transit light curves of GJ 3470b by considering plage occultation anomalies inside the transit for different values of wavelength. We found that the observed excess in the planet's radius in short wavelengths can be explained by the overlap of this planet with a plage with a filling factor of $2.56 \%$ and temperature contrast of $100 \mathrm{~K}$ (Fig. 5). The size and relative brightness of such a plage results in photometric variations of about $1.3 \%$ at $786 \mathrm{~nm}$ wavelength, which is compatible with the observed value $1 \%$.

In larger wavelengths, however, our plage occultation scenario was not able to properly model the results reported by Nascimbeni et al. (2013) (at the wavelength of $963 \mathrm{~nm}$ ) and the values reported by Ehrenreich et al. (2014) for wavelengths between 1170 and $1650 \mathrm{~nm}$. One explanation is that the obtained planet radius was systemically offset toward a large value causing no significant decrements to be observed in the planet radius in the range of $963-1650 \mathrm{~nm}$ during two successive transits of the planet at $4520 \mathrm{~nm}$ detected by Demory et al. (2013). To examine this possibility, we assumed that the actual planet-tostar radius ratio is smaller than the reported value $(0.077)$ and

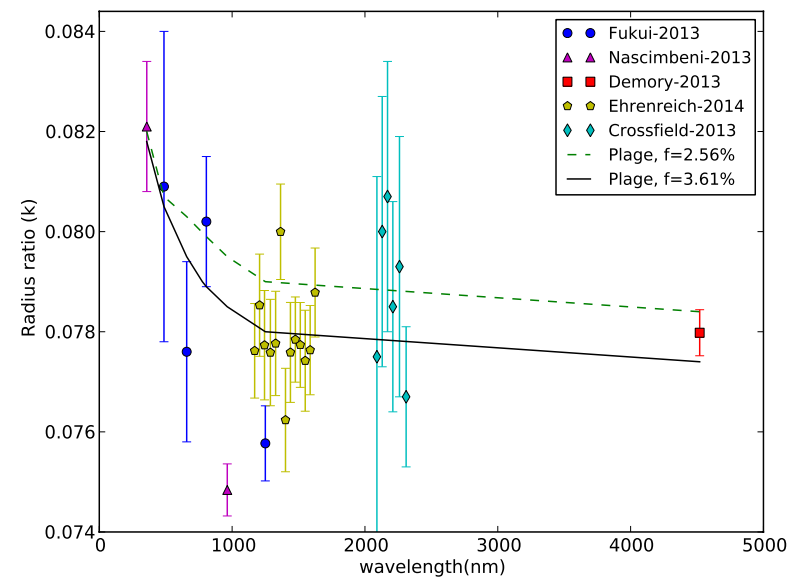

Fig. 5. Observed transmission spectrum of GJ $3470 \mathrm{~b}$ and reconstructed transmission spectrum by assuming the planet overlapping a stellar plage. The excess in the estimate of the planet radius-ratio in the blue side of spectrum can be reproduced by presuming a plage on the surface of GJ 3470. See text for more details.

obtained in this case, the minimum required plage's filling factor is $\sim 3.61 \%$ with a temperature contrast of $100 \mathrm{~K}$ (Fig. 5).

We note that these values for the plage's temperature and filling factor strongly depend on the choice of the temperature contrast. For instance, if we use a temperature ratio of GJ 3470 to that of the Sun $(\sim 0.62)$ to estimate the temperature contrast of a plage on the surface of GJ $3470(\sim 45 \mathrm{~K})$, the plage's filling factors of $2.56 \%$ and $3.61 \%$ increase to $7.29 \%$ and $9 \%$, respectively.

\section{Discussion and conclusions}

For the first time, we present the results of a study on the effect of the occultation of a stellar plages and spots on the transmission spectroscopy measurements of a planet. We carried out simulations by considering transiting systems with $\mathrm{G}$ or M stars, Jupiter- or Neptune-sized planets, and different values of a spot/plage's filling factor in different wavelength. Results indicated that there could be significant underestimation or overestimation of the planet-to-star radius ratio as a function of the wavelength. The maximum overestimation of the planet radius (10\%) may occur for the occultation of a plage by a planet that transits an M-dwarf in the short wavelength regime. Application of our calculations to the systems of the stars, HD 189733 and GJ 3470, indicated that the transmission spectroscopy measurements of the planets of these stars, and especially the reported excess in their planet-to-star radius ratio in the bluer part of the spectra, which were interpreted as the signature of blue sky, can almost exactly be reproduced by assuming that the planet occults a plage on the surface of these stars.

The results of our study strongly suggest that it is crucially important to rule out the possible contamination in the measurements due to effects of stellar activities (both occulted and nonocculted active regions) prior to interpreting the values obtained for the radius of a transiting planet in different wavelengths and attempting to set constrains on the planet's atmospheric models. The best strategy for doing so is to carry out several observations of a transit in a given wavelength and use the variations of transit depth as a function of time to assess the impact of potential stellar spot/plage occultation. In case of a very active star, which always harbors stellar spots or plages, all transits could be affected by the occultation. In that case, the alternative strategy would be to carry out simultaneous multiband photometric observations 


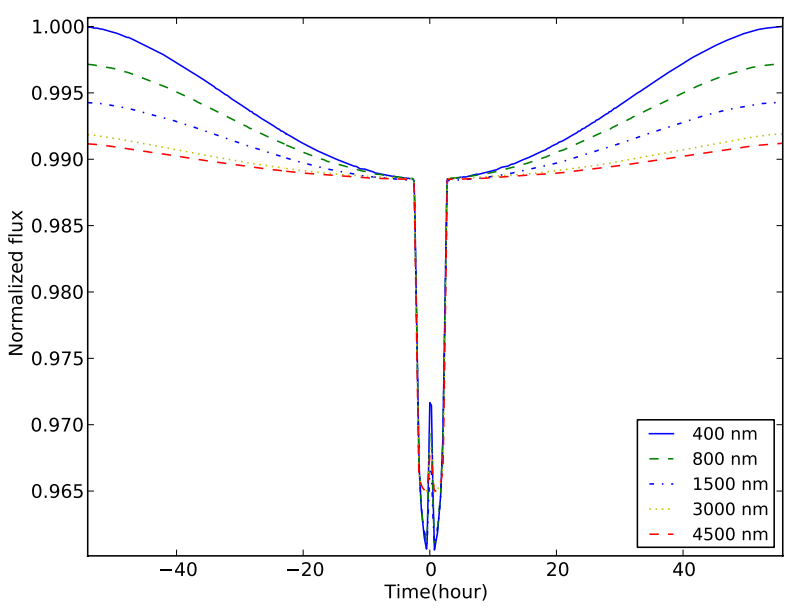

Fig. 6. Out-of-transit light curves for different wavelengths in a system with a stellar spot.

for several hours, which can correspond to half of the stellar rotation period before and after the transit. These observations will allow us to diagnose the presence of stellar spots/plages during the transit and can also provide information about the parameters of these regions, such as their sizes and temperature contrasts. Figure 6 shows an example of the distinct behavior of an outof-transit light curve in different wavelengths in the present of a stellar spot. The system depicted here consists of a Jupiter-sized planet with $R_{\mathrm{p}} / R_{*}=0.15$, which orbits in a 3-day orbit around an M-dwarf. The M-dwarf has a rotation period of 9 days and harbors a spot with $1 \%$ filling factor.

We note that the results presented here are based on simple assumptions. For instance, we always assumed that the transiting planet would occult the same plage at each epoch. This could be true in the case of a very active star, such as HD 189733. Our simulations were done by assuming a circular spot and plage which might affect the determination of spot/plage's filling factor. We also considered a constant temperature contrast of $100 \mathrm{~K}$ between a plage and its surrounding region on the star's photosphere. However, this value could be different for different stars. Larger or smaller temperature contrasts will result in lower or higher values for the filling factor. Finally, we assumed that the plage occultation anomalies appear in the middle of transit light curve, so that it would have the maximum impact on planet radius estimations. However, if these anomalies appear in other locations in the transit light curve, different values of the filling factor and/or plage-photosphere temperature contrast are required to account for the observed transmission spectroscopic properties of the system.
Acknowledgements. We would like to thank the anonymous referee for insightful comments that led to improvements in this paper. We acknowledge the support from the European Research Council/European Community under the FP7 through Starting Grant agreement number 239953, and by Fundação para a Ciência e a Tecnologia (FCT) in the form of grants reference SFRH/BD/51981/2012. N.C.S. also acknowledges the support from FCT through program Ciência 2007 funded by FCT/MCTES (Portugal) and POPH/FSE (EC). P.F. acknowledges support by Fundação para a Ciência e a Tecnologia (FCT) through the Investigador FCT contract of reference IF/01037/2013 and POPH/FSE (EC) by FEDER funding through the program "Programa Operacional de Factores de Competitividade - COMPETE". This work has been carried out within the frame of the National Center for Competence in Research PlanetS supported by the Swiss National Science Foundation (SNSF). D.E. acknowledges the financial support of the SNSF N.H. acknowledges support from NASA Origins program through grant NNX12AQ62G and NASA ADAP program through grant NNX13AF20G.

\section{References}

Berdyugina, S. V. 2005, Liv. Rev. Sol. Phys., 2, 8

Boisse, I., Moutou, C., Vidal-Madjar, A., et al. 2009, A\&A, 495, 959

Bonfils, X., Gillon, M., Udry, S., et al. 2012, A\&A, 546, A27

Brown, T. M., Charbonneau, D., Gilliland, R. L., Noyes, R. W., \& Burrows, A. 2001, ApJ, 552, 699

Charbonneau, D., Brown, T. M., Noyes, R. W., \& Gilliland, R. L. 2002, ApJ, 568,377

Claret, A., \& Bloemen, S. 2011, VizieR Online Data Catalog, 352, 99075

Crossfield, I. J. M., Barman, T., Hansen, B. M. S., \& Howard, A. W. 2013, A\&A, 559, A33

Czesla, S., Huber, K. F., Wolter, U., Schröter, S., \& Schmitt, J. H. M. M. 2009, A\&A, 505, 1277

Demory, B.-O., Torres, G., Neves, V., et al. 2013, ApJ, 768, 154

Désert, J.-M., Vidal-Madjar, A., Lecavelier Des Etangs, A., et al. 2008, A\&A, 492, 585

Ehrenreich, D., Hébrard, G., Lecavelier des Etangs, A., et al. 2007, ApJ, 668, L179

Ehrenreich, D., Bonfils, X., Lovis, C., et al. 2014, A\&A, in press, DOI: $10.1051 / 0004-6361 / 201423809$

Fukui, A., Narita, N., Kurosaki, K., et al. 2013, ApJ, 770, 95

Knutson, H. A., Howard, A. W., \& Isaacson, H. 2010, ApJ, 720, 1569

Knutson, H. A., Madhusudhan, N., Cowan, N. B., et al. 2011, ApJ, 735, 27

Kreidberg, L., Bean, J. L., Désert, J.-M., et al. 2014, Nature, 505, 69

Lecavelier Des Etangs, A., Pont, F., Vidal-Madjar, A., \& Sing, D. 2008, A\&A, 481, L83

Meunier, N., Desort, M., \& Lagrange, A.-M. 2010, A\&A, 512, A39

Nascimbeni, V., Piotto, G., Pagano, I., et al. 2013, A\&A, 559, A32

Oshagh, M., Santos, N. C., Boisse, I., et al. 2013a, A\&A, 556, A19

Oshagh, M., Boisse, I., Boué, G., et al. 2013b, A\&A, 549, A35

Pont, F., Gilliland, R. L., Moutou, C., et al. 2007, A\&A, 476, 1347

Pont, F., Knutson, H., Gilliland, R. L., Moutou, C., \& Charbonneau, D. 2008, MNRAS, 385, 109

Pont, F., Sing, D. K., Gibson, N. P., et al. 2013, MNRAS, 432, 2917

Poppenhaeger, K., Schmitt, J. H. M. M., \& Wolk, S. J. 2013, ApJ, 773, 62

Seager, S., \& Sasselov, D. D. 2000, ApJ, 537, 916

Sing, D. K., Désert, J.-M., Lecavelier Des Etangs, A., et al. 2009, A\&A, 505, 891

Sing, D. K., Pont, F., Aigrain, S., et al. 2011, MNRAS, 416, 1443

Solanki, S. K. 2003, A\&ARv, 11, 153

Unruh, Y. C., Solanki, S. K., \& Fligge, M. 1999, A\&A, 345, 635

Worden, J. R., White, O. R., \& Woods, T. N. 1998, ApJ, 496, 998 